\title{
COMPLETE GROUPS WITH NONABELIAN COMPOSITION FACTORS
}

\author{
JAY ZIMMERMAN
}

\begin{abstract}
A finite group is said to be complete if it has trivial center and if every automorphism is an inner automorphism. A finite group with nonabelian composition factors has a unique completely reducible radical (CR radical). We consider finite groups with nonabelian composition factors whose CR radical consists of complete simple groups and we give necessary and sufficient conditions for such a group to be complete. This involves finding group theoretic conditions which are necessary and sufficient for a finite centerless group to occur as a self-normalizing subgroup of a direct product of symmetric groups.
\end{abstract}

1. Introduction. A finite group is called semisimple if it contains no nontrivial abelian normal subgroups. A completely reducible group is a group which is isomorphic to a direct product of simple groups. It is well known that a finite semisimple group $G$ contains a unique maximal normal completely reducible group $R$, called its completely reducible radical. It is easily seen that this completely reducible radical is characteristic in the whole group (Robinson $[1$, p. 85]). Hence, we have that $R \leq G \leq$ Aut $R$ under the obvious embeddings; and this characterizes all semisimple groups with completely reducible radical $R$. It is an elementary exercise to show that in this situation Aut $G \cong N_{\text {Aut } R}(G)$ where we are identifying $G$ with a subgroup of Aut $R$ in a natural way. In particular, if $G$ is complete, then $G \cong N_{\text {Aut } R}(G)$. Conversely, a self-normalizing subgroup of Aut $R$ containing $R$ is complete. The above discussion leads to the following proposition (see Robinson $[1$, p. 86]).

PROPOSITION 1. There is a bijection between isomorphism classes of finite complete semisimple groups with radical $R$ and conjugacy classes of self-normalizing subgroups of Out $R$.

This follows easily from the above comments and the canonical homomorphism Aut $R \rightarrow$ Out $R$.

We will be primarily concerned with those finite groups whose composition factors are all nonabelian. We will call these groups purely nonabelian or p.n.a. groups. If $R \cong \operatorname{Dr}_{i=1}^{m}\left(\operatorname{Dr}_{j=1}^{n_{i}} S_{i}\right)$ where the $S_{i}$ are nonisomorphic nonabelian finite simple groups, then

$$
\text { Out } R \cong \operatorname{Dr}_{i=1}^{m}\left(\left(\operatorname{Out} S_{i}\right) \operatorname{wr}\left(\operatorname{Sym}\left(n_{i}\right)\right)\right) .
$$

Received by the editors October 20, 1985. Presented to the Society, January 9, 1986 at the New Orleans meeting.

1980 Mathematics Subject Classification (1985 Revision). Primary 20B35, 20F28.

Key words and phrases. Complete group, permutation group, symmetric group. 
The Schreier Conjecture, which has been verified on a case by case basis, asserts that Out $S$ is solvable for all nonabelian finite simple groups $S$. Suppose that some Out $S_{i}$ has nontrivial center and let $1 \neq x \in Z\left(\right.$ Out $\left.S_{i}\right)$. Define $((x, \ldots, x), 1)=\bar{x}$ as an element of $\left(\left(\operatorname{Out} S_{i}\right) \mathrm{wr}\left(\operatorname{Sym}\left(n_{i}\right)\right)\right)$. We may consider $\bar{x}$ as an element of Out $R$, and hence $\bar{x} \in Z($ Out $R)$. However, this implies that Out $R$ has no selfnormalizing p.n.a. subgroups. We conclude that if Out $R$ contains a self-normalizing p.n.a. subgroup, then $Z$ (Out $\left.S_{i}\right)=1$ for all $i$. This leads naturally to the following conjecture.

CONJECTURE. If $H$ is a complete p.n.a. group, then all simple direct factors of the completely reducible radical of $H$ are complete.

The status of this conjecture is unknown; but it leads us to the consideration of self-normalizing p.n.a. subgroups of Out $R$ where every simple direct factor of $R$ is complete. In this case, Out $R \cong \operatorname{Dr}_{i=1}^{m} \operatorname{Sym}\left(n_{i}\right)$ and we have to determine all self-normalizing p.n.a. subgroups of a direct product of symmetric groups.

2. Normalizers of permutation groups. We will now begin by proving a general theorem which gives conditions for an abstract group with trivial center to occur as a self-normalizing subgroup of a direct product of symmetric groups. Subsequently, we will exhibit several techniques which generate a large number of examples of self-normalizing p.n.a. subgroups of symmetric groups.

We will use these examples to disprove some further conjectures. For example, we will obtain a complete p.n.a. group whose composition series contains simple factors which are not complete. However, this example does not settle the above conjecture because the factor is not contained in the completely reducible radical.

We will begin by examining the situation for a single symmetric group $\operatorname{Sym}(X)$.

DEFINITION. Let $G$ be a group and let $\overline{(H, e)}=\left\{\left(H_{i}, e_{i}\right) \mid 1 \leq i \leq k\right\}$ be a set of ordered pairs consisting of a subgroup $H_{i}$ and $G$ and a positive integer $e_{i}$, called the multiplicity of $H_{i}$. Suppose that the $H_{i}$ are not $G$-conjugate. Define Aut $(G \mid \overline{(H, e)})$ to be the subgroup of Aut $G$ consisting of those automorphisms $\varphi$ which satisfy the following properties:

(1) $\varphi$ permutes the conjugates of $H_{1}, \ldots, H_{k}$, say $H_{i}^{\varphi}=H_{(i) \pi}^{u_{i}}$ where $\pi \in \operatorname{Sym}(k)$ and $u_{i} \in G$.

(2) $e_{i}=e_{(i) \pi}$ for all $i$.

Furthermore, if all of the $e_{i}$ are equal to one then we will write $\operatorname{Aut}(G \mid \bar{H})$ in place of $\operatorname{Aut}(G \mid \overline{(H, e)})$.

THEOREM 2. Let $G$ be a subgroup of $S=\operatorname{Sym}(X)$. Let $H_{1}, \ldots, H_{k}$ be a complete set of nonconjugate stabilizers of elements of $X$ in $G$. Define the multiplicity $e_{i}$ of $H_{i}$ to be the number of $G$-orbits whose stabilizers are conjugate to $H_{i}$ in $G$. Then there is a split exact sequence

$$
\underset{i=1}{k}\left(\left(N_{G}\left(H_{i}\right) / H_{i}\right) \text { wr }\left(\operatorname{Sym}\left(e_{i}\right)\right)\right) \nrightarrow N_{S}(G) \rightarrow \operatorname{Aut}(G \mid \overline{(H, e)}) .
$$

ProOF. Let $\left\{O_{i j} \mid 1 \leq i \leq k, 1 \leq j \leq e_{i}\right\}$ be the set of all $G$-orbits of $X$ labeled so that the set $S_{i}=\left\{\operatorname{St}_{G}(x) \mid x \in \bigcup_{l=1}^{e_{i}} O_{i l}\right\}$ consists of all stabilizers that are $G$-conjugate to $H_{i}$. Notice that if $x \in X$ and $\sigma \in N_{S}(G)$, then $\operatorname{St}_{G}((x) \sigma)=$ $\sigma^{-1}\left(\mathrm{St}_{G}(x)\right) \sigma$. It follows that we may choose an element $a(i, j)$ from each orbit $O_{i j}$ such that $\operatorname{St}_{G}(a(i, j))=H_{i}$ for $1 \leq j \leq e_{i}$. 
Let $\sigma \in N_{S}(G)$. Since the stabilizer of any element in $O_{i j}$ is conjugate to $H_{i}$ for $1 \leq j \leq e_{i}$, the stabilizer of any element of $O_{i j} \sigma$ is conjugate to $H_{i}^{\sigma}=H_{(i) \pi}^{u_{i}}$ where $\pi \in \operatorname{Sym}(k)$ and $u_{i} \in G$. It follows that $e_{i}=e_{(i) \pi}$ and hence $O_{i j} \sigma=O_{(i) \pi,(j) \tau_{i}}$ where $\pi \in \operatorname{Sym}(k)$ and $\tau_{i} \in \operatorname{Sym}\left(e_{i}\right)$. We deduce that there exists an element $u(i, j) \in G$ such that $a(i, j) \sigma=a\left((i) \pi,(j) \tau_{i}\right) u(i, j)$. The element $u(i, j)$ could be replaced by any element of the coset $\left(H_{(i) \pi} u(i, j)\right)$. We have just shown that we may associate with any $\sigma \in N_{S}(G)$ the permutations $\pi \in \operatorname{Sym}(k), \tau_{i} \in \operatorname{Sym}\left(e_{i}\right)$ and the cosets $\left\{\left(H_{(i) \pi} u(i, j)\right) \mid 1 \leq i \leq k, 1 \leq j \leq e_{i}\right\}$.

Since $\sigma$ normalizes $G$, it induces an automorphism $\varphi$ of $G$ by conjugation. We will show that $\varphi$ is an element of $\operatorname{Aut}(G \mid \overline{(H, e)})$. For any $g \in G$, consider the equations

$$
(a(i, j) g) \sigma=(a(i, j) \sigma) g^{\sigma}=\left(a\left((i) \pi,(j) \tau_{i}\right) u(i, j)\right) g^{\varphi} .
$$

If we choose $g \in H_{i}=\operatorname{St}_{G}(a(i, j))$, then we can deduce

$$
\left(a\left((i) \pi,(j) \tau_{i}\right) u(i, j)\right)=(a(i, j) \sigma)=(a(i, j) g) \sigma=\left(a\left((i) \pi,(j) \tau_{i}\right) u(i, j)\right) g^{\varphi} .
$$

This implies that $g^{\varphi}$ stabilizes $\left(a\left((i) \pi,(j) \tau_{i}\right) u(i, j)\right)$ and hence

$$
g^{\varphi} \in\left(\operatorname{St}_{G}\left(a\left((i) \pi,(j) \tau_{i}\right)\right)\right)^{u(i, j)} .
$$

It follows that if $g \in H_{i}$, then $g^{\varphi} \in H_{(i) \pi}^{u(i, j)}$ and hence $H_{i}^{\varphi}=H_{(i) \pi}^{u(i, j)}$. Since $e_{i}=e_{(i) \pi}$, this shows that $\varphi \in \operatorname{Aut}(G \mid \overline{(H, e)})$.

In summary, to every $\sigma \in N_{S}(G)$ we may associate the quadruple

$$
\left(\varphi, \pi,\left(\tau_{i}\right)_{i=1}^{k},\left\{H_{(i) \pi} u(i, j) \mid 1 \leq i \leq k, 1 \leq j \leq e_{i}\right\}\right)
$$

where $\varphi \in \operatorname{Aut}(G \mid \overline{(H, e)}), \pi \in \operatorname{Sym}(k), \tau_{i} \in \operatorname{Sym}\left(e_{i}\right)$, and $e_{i}=e_{(i) \pi}$ for all $i$.

Conversely assume that we have such a quadruple. Every element of $X$ is expressible in the form $a(i, j) g$ for some $i, j$ and $g \in G$. Define $\sigma: X \rightarrow X$ by the rule

$$
(a(i, j) g) \sigma=\left(a\left((i) \pi,(j) \tau_{i}\right)\right) u(i, j) g^{\varphi}
$$

where $g \in G$. First, we must check that $\sigma$ is well defined. If $h \in H_{i}=\operatorname{St}_{G}(a(i, j))$, then $h^{\varphi}=(\bar{h})^{u(i, j)}$ for some $\bar{h} \in H_{(i) \pi}$. Then

$$
\begin{aligned}
(a(i, j) h g) \sigma & =\left(a\left((i) \pi,(j) \tau_{i}\right) u(i, j)\right)(h g)^{\varphi}=\left(a\left((i) \pi,(j) \tau_{i}\right) \bar{h} u(i, j)\right) g^{\varphi} \\
& =\left(a\left((i) \pi,(j) \tau_{i}\right) u(i, j)\right) g^{\varphi}=(a(i, j) g) \sigma .
\end{aligned}
$$

Next, the map $\sigma$ has an inverse, namely

$$
(a(i, j) g) \sigma^{-1}=a\left((i) \pi^{-1},(j) \tau_{(i) \pi^{-1}}^{-1}\right)\left(u\left((i) \pi^{-1},(j) \tau_{(i) \pi^{-1}}^{-1}\right)\right)^{-\varphi^{-1}} g^{\varphi^{-1}} .
$$

It follows that $\sigma \in S$.

We must now show that $\sigma \in N_{S}(G)$. Let $x, g \in G$. We compute that

$$
\begin{aligned}
(a(i, j) g) \sigma^{-1} x \sigma & \\
& =\left(\left(a\left((i) \pi^{-1},(j) \tau_{(i) \pi^{-1}}^{-1}\right)\left(u\left((i) \pi^{-1},(j) \tau_{(i) \pi^{-1}}^{-1}\right)\right)^{-\varphi^{-1}} g^{\varphi^{-1}}\right) x\right) \sigma \\
& =a(i, j) u\left((i) \pi^{-1},(j) \tau_{(i) \pi^{-1}}^{-1}\right)\left(\left(u\left((i) \pi^{-1},(j) \tau_{(i) \pi^{-1}}^{-1}\right)\right)^{-\varphi^{-1}} g^{\varphi^{-1}} x\right)^{\varphi} \\
& =(a(i, j) g) x^{\varphi} .
\end{aligned}
$$


It follows that $\sigma^{-1} x \sigma=x^{\varphi}$ and in particular $\sigma \in N_{S}(G)$. We have established a bijection between the quadruples $\left(\varphi, \pi,\left(\tau_{i}\right),\left(H_{(i) \pi} u(i, j)\right)\right)$ and the elements $\sigma$ of $N_{S}(G)$.

Now define the homomorphism $\Theta: N_{S}(G) \rightarrow$ Aut $G$ by $(\sigma) \Theta=\varphi$. It is clear that $\operatorname{Im} \Theta=\operatorname{Aut}(G \mid \overline{(H, e)})$.

We will now obtain an expression for $\operatorname{Ker} \Theta$. Let $\sigma \in \operatorname{Ker} \Theta$. Then $\varphi=1$ and hence $H_{i}=H_{(i) \pi}^{u(i, j)}$. This implies that $(i) \pi=i$ for all $i$ and therefore $\pi=1$. it also implies that $u(i, j) \in N_{G}\left(H_{i}\right)$. Thus under the above bijection, $\sigma$ corresponds to the quadruple $\left(1,1,\left(\tau_{i}\right),\left(H_{i} u(i, j)\right)\right)$ with $u(i, j) \in N_{G}\left(H_{i}\right)$. It is easily verified that the group operation on $N_{S}(G)$ induces the following operation on the set of all quadruples which correspond to elements of $\operatorname{Ker} \Theta$,

$$
\begin{gathered}
\left(1,1,\left(\tau_{i}^{\prime}\right),\left(H_{i} u^{\prime}(i, j)\right)\right) \cdot\left(1,1,\left(\tau_{i}^{\prime \prime}\right),\left(H_{i} u^{\prime \prime}(i, j)\right)\right) \\
=\left(1,1,\left(\tau_{i}^{\prime} \tau_{i}^{\prime \prime}\right),\left(H_{i} u^{\prime \prime}\left(i,(j) \tau_{i}^{\prime}\right) u^{\prime}(i, j)\right)\right) .
\end{gathered}
$$

It is obvious that $\operatorname{Ker} \Theta \cong \operatorname{Dr}_{i=1}^{k}\left(\left(N_{G}\left(H_{i}\right) / H_{i}\right)\right.$ wr $\left.\left(\operatorname{Sym}\left(e_{i}\right)\right)\right)$ by the map $\sigma \mapsto$ $\left(\tau_{i},\left(H_{i} u(i, j)\right)_{j}\right)_{i}$. Hence we have the short exact sequence

$$
\underset{i=1}{k}\left(\left(N_{G}\left(H_{i}\right) / H_{i}\right) \operatorname{wr}\left(\operatorname{Sym}\left(e_{i}\right)\right)\right) \nrightarrow N_{S}(G) \rightarrow \operatorname{Aut}(G \mid \overline{(H, e)}) .
$$

Finally, we must show that this sequence splits. Let $\varphi \in \operatorname{Aut}(G \mid \overline{(H, e)})$; then $H_{i}^{\varphi}=H_{(i) \pi}^{u_{i}}$ for some $\pi \in \operatorname{Sym}(k)$ and $u_{i} \in G$. Define $\nu: \operatorname{Aut}(G \mid \overline{(H, e)}) \rightarrow N_{S}(G)$ by $\nu: \varphi \mapsto\left(\varphi, \pi,(1),\left(H_{i} u_{i}\right)\right)=\sigma$. We will now show that $\nu$ is a homomorphism. Consider the elements $\left(\varphi_{1}\right) \nu$ and $\left(\varphi_{2}\right) \nu$. As above there exist $\pi_{1}, \pi_{2} \in \operatorname{Sym}(k)$, $u_{i}^{1}, u_{i}^{2} \in G$ and $\varphi_{1}, \varphi_{2} \in \operatorname{Aut}(G \mid \overline{(H, e)})$ such that

$$
(a(i, j) g)\left(\left(\varphi_{l}\right) \nu\right)=a\left((i) \pi_{l}, j\right) u_{i}^{l} g^{\varphi_{l}}
$$

for $l=1$ or 2 . It follows that

$$
H_{i}^{\varphi_{1} \varphi_{2}}=\left(H_{(i) \pi_{1}}^{u_{i}^{1}}\right)^{\varphi_{2}}=H_{(i) \pi_{1} \pi_{2}}^{u_{i}^{2}\left(u_{i}^{1}\right)^{\varphi_{2}}}
$$

and hence

$$
\begin{aligned}
& (a(i, j) g)\left(\left(\varphi_{1}\right) \nu\left(\varphi_{2}\right) \nu\right)=\left(a\left((i) \pi_{1}, j\right) u_{i}^{1} g^{\varphi_{1}}\right)\left(\varphi_{2}\right) \nu \\
& \quad=a\left((i) \pi_{1} \pi_{2}, j\right) u_{i}^{2}\left(u_{i}^{1}\right)^{\varphi_{2}} g^{\varphi_{1} \varphi_{2}}=(a(i, j) g)\left(\varphi_{1} \varphi_{2}\right) \nu .
\end{aligned}
$$

This shows that $\nu$ is a homomorphism and hence it is easily seen to be a splitting.

Suppose that $G$ is a self-normalizing subgroup of $S=\operatorname{Sym}(X)$ with trivial center. Theorem 2 shows that $\operatorname{Aut}(G \mid \overline{(H, e)})=\operatorname{Inn} G$. Since $Z(G)=1$, we see that $\operatorname{Aut}(G \mid \overline{(H, e)}) \cong G$ and the map $N_{S}(G) \rightarrow \operatorname{Aut}(G \mid \overline{(H, e)})$ is an isomorphism. Since $\operatorname{Dr}_{i=1}^{k}\left(\left(N_{G}\left(H_{i}\right) / H_{i}\right)\right.$ wr $\left.\left(\operatorname{Sym}\left(e_{i}\right)\right)\right)=1$, we also have that $H_{i}=N_{G}\left(H_{i}\right)$ and $e_{i}=1$ for all $i$.

We can now characterize the self-normalizing subgroups of a symmetric group which have trivial center.

THEOREM 3. Let $G$ be an abstract finite group with trivial center. Then $G$ is isomorphic with a self-normalizing subgroup of some finite symmetric group if and only if there exist subgroups $H_{1}, \ldots, H_{k}$ of $G$ satisfying the conditions:

(i) No two of $H_{1}, \ldots, H_{k}$ are conjugate in $G$. 
(ii) $N_{G}\left(H_{i}\right)=H_{i}$ for all $i$.

(iii) $\bigcap_{i=1}^{k}\left(\bigcap_{g \in G} g^{-1} H_{i} g\right)=1$.

(iv) $\operatorname{Aut}(G \mid \bar{H})=\operatorname{Inn} G$.

PROOF. We have already proven that conditions (i), (ii), and (iv) are necessary. Since $\bigcap_{i=1}^{k}\left(\bigcap_{g \in G} g^{-1} H_{i} g\right)$ is the set of elements of $G$ which stablilize every element of $X$, it must be trivial. Thus condition (iii) is also necessary.

Conversely assume that such a set of subgroups exists. Define $X$ to be the set of all right cosets of the $H_{i}$ and let $G$ act on $X$ by right multiplication. If $h$ acts trivially on all of $X$, then $h \in \bigcap_{i=1}^{k}\left(\bigcap_{g \in G} g^{-1} H_{i} g\right)$. It follows that $G$ acts faithfully on $X$ by condition (iii). We can see that the $G$-orbits are $\left\{H_{i} g \mid g \in G\right\}$ for all $i$ and $\operatorname{St}_{G}\left(H_{i} g\right)=\left(\operatorname{St}_{G}\left(H_{i}\right)\right)^{g}=H_{i}^{g}$. If $H_{i}^{g_{1}}=H_{j}^{g_{2}}$, then by condition (i), the indices $i$ and $j$ are equal. It follows that distinct orbits have nonconjugate stabilizers. Hence if we regard $G$ as a subgroup of $\operatorname{Sym}(X)$, we have that $e_{i}=1$ in the notation of Theorem 2. We may apply Theorem 2 to show that $N_{\mathrm{Sym}(X)}(G)=G$.

Now we will consider the more general case where we have a direct product of symmetric groups. Let $X$ be a disjoint union of nonempty subsets $X_{i}$, symbolically $X=X_{1} \dot{\cup} \cdots \dot{\cup} X_{l}$. Let $G$ be a group of permutations of $X$ which fix each $X_{i}$ as a set. Thus

$$
G \leq S_{0}=\operatorname{Sym}\left(X_{1}\right) \times \cdots \times \operatorname{Sym}\left(X_{l}\right) \leq S=\operatorname{Sym}(X) .
$$

First, we would like to identify $N_{S_{0}}(G)$, which is, of course, equal to $S_{0} \cap N_{S}(G)$. We will use the characterization of $N_{S}(G)$ developed in Theorem 2 and try to find out when $\sigma \in N_{S}(G)$ is also in $S_{0}$.

Let $\sigma=\left(\varphi, \pi,\left(\tau_{i}\right),\left(H_{(i) \pi} u(i, j)\right)\right) \in N_{S}(G)$. If $\sigma \in S_{0}$, then both $a(i, j)$ and $a\left((i) \pi,(j) \tau_{i}\right)=(a(i, j) \sigma)(u(i, j))^{-1}$ must be in the same set $X_{t}$ for some $t$. It follows that if $H_{i}$ is a stabilizer associated with an orbit contained in $X_{t}$, then so is $H_{i}^{\varphi}$. Hence $\varphi$ must permute conjugates of the $H_{i}$ that are associated with the same $X_{t}$. We shall write the subgroup of all $\varphi$ in $\operatorname{Aut}(G \mid \overline{(H, e)})$ with this property as $\operatorname{Aut}(G|\overline{(H, e)}| \bar{X})$ where $\bar{X}=\left\{X_{1}, \ldots, X_{l}\right\}$. If $e_{i}=1$, then we will write $\operatorname{Aut}(G|\bar{H}| \bar{X})$.

Let $A$ be the semidirect product

$$
\operatorname{Aut}(G|\overline{(H, e)}| \bar{X}) \ltimes \underset{i=1}{k}\left(\operatorname{Sym}\left(e_{i}\right)\right)
$$

where $\varphi$ in $\operatorname{Aut}(G|\overline{(H, e)}| \bar{X})$ acts on $\tau$ in $\operatorname{Dr}_{i=1}^{k}\left(\operatorname{Sym}\left(e_{i}\right)\right)$ according to $\left(\tau^{\varphi}\right)_{i}=$ $\tau_{(i) \pi^{-1}}$. Define $\theta: N_{S}(G) \rightarrow A$ by

$$
\theta:\left(\varphi, \pi,\left(\tau_{i}\right),\left(H_{(i) \pi} u(i, j)\right)\right) \mapsto\left(\varphi,\left(\tau_{i}\right)_{1 \leq i \leq k}\right) .
$$

The following equation shows that $\theta$ is a homomorphism:

$$
[(\sigma) \theta]\left[\left(\sigma^{\prime}\right) \theta\right]=\left(\varphi \varphi^{\prime}, \tau_{i}^{\varphi^{\prime}} \tau_{i}^{\prime}\right)=\left(\varphi \varphi^{\prime}, \tau_{\left((i) \pi^{\prime-1}\right)} \tau_{i}^{\prime}\right)=\left(\sigma \sigma^{\prime}\right) \theta .
$$

Notice that $\operatorname{Ker} \theta$ consists of all $\sigma \in N_{S}(G)$ which correspond to $\left.\left(1,1,(1), H_{i} u(i, j)\right)\right)$. Thus we get the following short exact sequence:

$$
\underset{i=1}{\stackrel{k}{\operatorname{Dr}}}\left(\underset{j=1}{\operatorname{Pr}_{i}} N\left(H_{i}\right) / H_{i}\right) \nrightarrow N_{S}(G) \stackrel{\theta}{\rightarrow} A
$$


Let $V=\left(N_{S_{0}}(G)\right) \theta$. If $\sigma \in \operatorname{Ker} \theta$, then $a(i, j) \sigma=a(i, j) u(i, j)$ and so Ker $\theta \leq S_{0}$. This yields the following short exact sequence:

$$
\underset{i=1}{k}\left({\underset{\operatorname{Dr}}{\operatorname{Dr}_{i}}}_{y=1} N_{G}\left(H_{i}\right) / H_{i}\right) \longmapsto N_{S_{0}}(G) \rightarrow V
$$

We will show that the composite of the canonical maps

$$
N_{S_{0}}(G) \rightarrow V \rightarrow \operatorname{Aut}(G|\overline{(H, e)}| \bar{X})
$$

is surjective. Now suppose that $N_{S_{0}}(G)=G$, then $N_{G}\left(H_{i}\right)=H_{i}$. If $\varphi \in$ $\operatorname{Aut}(G|\overline{(H, e)}| \bar{X})$ then for each $j$ from 1 to $e_{i}$, there exists $j^{\prime}$ between 1 and $e_{i}$ such that $a(i, j)$ and $a\left((i) \pi, j^{\prime}\right)$ are in the same set $X_{s}$ where $H_{i}^{\varphi}=H_{(i) \pi}^{u(i, j)}$. Define $\tau_{i} \in \operatorname{Sym}\left(e_{i}\right)$ by $(j) \tau_{i}=j^{\prime}$. Define $\sigma=\left(\varphi, \pi,\left(\tau_{i}\right),\left(H_{(i) \pi} u(i, j)\right)\right)$ where all quantities are as defined above. We can deduce that $\sigma \in N_{S_{0}}(G)$ and $(\sigma) \theta=\left(\left(\tau_{i}\right), \varphi\right)$. It follows that the canonical projection of $V$ into $\operatorname{Aut}(G|\overline{(H, e)}| \bar{X})$ is surjective. It is also clear that $\langle((1), \varphi) \mid \varphi \in \operatorname{Inn} G\rangle \leq V$. Therefore, if $N_{S_{0}}(G)=G$, then $e_{i}=1$ for all $i$ and $\operatorname{Aut}(G|\bar{H}| \bar{X})=\operatorname{Inn} G$.

These facts show the necessity of the hypothesis in the following theorem.

THEOREM 4. Let $G$ be an abstract finite group with trivial center. Then $G$ is isomorphic with a self-normalizing subgroup of a direct product of l finite symmetric groups if and only if there exist subgroups $H_{1}, \ldots, H_{k}$ and a partition $Y_{1} \dot{\cup} \cdots \dot{\cup} Y_{l}$ of the set $Y=\left\{H_{i} g \mid g \in G, 1 \leq i \leq k\right\}$ satisfying the conditions:

(i) No two of $H_{1}, \ldots, H_{k}$ are conjugate on $G$.

(ii) $N_{G}\left(H_{i}\right)=H_{i}$ for all $i$.

(iii) $\bigcap_{i=1}^{k}\left(\bigcap_{g \in G} g^{-1} H_{i} g\right)=1$.

(iv) Each set $Y_{i}$ is the union of all right cosets of certain of the subgroups $H_{j}$.

(v) $\operatorname{Aut}(G|\bar{H}| \bar{Y})=\operatorname{Inn} G$.

ProOF. Assume that $G$ is a self-normalizing subgroup of $\operatorname{Sym}\left(X_{i}\right) \times \cdots \times$ $\operatorname{Sym}\left(X_{l}\right)$. Define the $H_{i}$ as $\operatorname{St}_{G}(a(i, j))$ as before. We have already shown that $e_{i}=1$ for all $i, N_{G}\left(H_{i}\right)=H_{i}$, and $\operatorname{Aut}(G|\bar{H}| \bar{X})=\operatorname{Inn} G$. Define $Y_{r}$ as the set of all right cosets of every $H_{i}$ that is the stabilizer associated with an orbit contained in $X_{r}$. It is clear that all five conditions are satisfied.

Conversely, assume that we have the groups $H_{1}, \ldots, H_{k}$ and the partition $Y_{1}, \ldots$, $Y_{l}$ of $Y$ satisfying all of the given conditions. We can embed $G$ in $S_{0}=\operatorname{Dr}_{i=1}^{k} \operatorname{Sym}\left(Y_{i}\right)$ in the obvious way and it follows easily from conditions (ii) and (v) that $N_{S_{0}}(G)=$ G.

The purpose of classifying self-normalizing p.n.a. subgroups of a direct product of symmetric groups was to decide which p.n.a. groups were complete. Therefore, let us recall how we can construct a complete group from a self-normalizing p.n.a. subgroup of a direct product of symmetric groups. Let $H \leq \operatorname{Sym}\left(n_{1}\right) \times \cdots \times \operatorname{Sym}\left(n_{l}\right)$ be a self-normalizing p.n.a. subgroup. Let $S_{1}, \ldots, S_{l}$ be a set of nonisomorphic complete nonabelian finite simple groups. Define

$$
R=\operatorname{Dr}_{i=1}^{l}\left({\underset{j=1}{n_{i}}}_{j=1} S_{i}\right)
$$

It follows that

$$
\text { Out } R \cong \operatorname{Sym}\left(n_{1}\right) \times \cdots \times \operatorname{Sym}\left(n_{l}\right) \text {. }
$$


The preimage $G$ of $H$ under Aut $R \rightarrow \operatorname{Sym}\left(n_{1}\right) \times \cdots \times \operatorname{Sym}\left(n_{l}\right)$ is a complete p.n.a. group. Moreover, if the conjecture at the beginning of this section is correct, then this construction will give all complete p.n.a. groups.

3. Examples. In the remainder of this section we shall give explicit examples of self-normalizing p.n.a. subgroups of a symmetric group. The simplest examples arise as follows. Let $G$ be a complete nonabelian finite simple group and let $H$ be a maximal subgroup of $G$. The conditions of Theorem 3 are all satisfied with $k=1$ and $H_{1}=H$. Hence $G$ can be embedded in $\operatorname{Sym}(|G: H|)$ as a self-normalizing subgroup. In particular, the Mathieu groups $M_{11}, M_{23}$ and $M_{24}$ may be embedded in $\operatorname{Sym}(11), \operatorname{Sym}(23)$ and $\operatorname{Sym}(24)$ respectively as self-normalizing subgroups. Thus we obtain as complete p.n.a. groups the permutational wreath products $S$ wr $M_{11}$, $S$ wr $M_{23}$ and $S$ wr $M_{24}$ where $S$ is a complete simple group.

We will begin with a technique for constructing self-normalizing p.n.a. subgroups of a symmetric group given a proper p.n.a. subgroup of a directly indecomposable p.n.a. group. If $H$ is a subgroup of $G$, then define ${ }^{*} H$ and $H^{*}$ as the images of $H$ under the left and right regular representations of $G$ respectively.

LEMMA 5. Let $H$ be a proper subgroup with trivial center of a finite directly indecomposable group $G$ with $Z(G)=1$. If $N_{\mathrm{Aut} G}(H)=\operatorname{Inn} H$, then $\left({ }^{*} H \times G^{*}\right)$ is self-normalizing in $\operatorname{Sym}(G)$.

PrOOF. Let $\gamma \in N_{\mathrm{Sym}(G)}\left({ }^{*} H \times G^{*}\right)$. It follows that

$$
\left({ }^{*} H \times G^{*}\right)=\left({ }^{*} H \times G^{*}\right)^{\gamma}=\left({ }^{*} H\right)^{\gamma} \times\left(G^{*}\right)^{\gamma} .
$$

The Remak-Krull-Schmidt Theorem implies that there exists a central automorphism $\alpha$ of $\left({ }^{*} H \times G^{*}\right)$ such that $\left({ }^{*} H\right)^{\gamma}=\left({ }^{*} H\right)^{\alpha}$ and $\left(G^{*}\right)^{\gamma}=\left(G^{*}\right)^{\alpha}$ (Robinson [1, p. 81]). Since $Z\left({ }^{*} H \times G^{*}\right)=1$, the automorphism $\alpha$ centralizes both $G^{*}$ and ${ }^{*} H$ and hence $\left({ }^{*} H\right)^{\gamma}=\left({ }^{*} H\right)$ and $\left(G^{*}\right)^{\gamma}=\left(G^{*}\right)$. The holomorph $\operatorname{Hol}(G)$ is defined to be $\left\langle\right.$ Aut $\left.G, G^{*}\right\rangle \leq \operatorname{Sym}(G)$, and it is easily shown to be equal to $N_{\operatorname{Sym}(G)}\left(G^{*}\right)$. It follows that by multiplying $\gamma$ by an element of $G^{*}$, we may construct $\bar{\gamma} \in$ Aut $G$ such that $\bar{\gamma}$ normalizes both $\left({ }^{*} H\right)$ and $\left(G^{*}\right)$. Now $\left({ }^{*} h\right) \bar{\gamma}={ }^{*}\left(h^{\bar{\gamma}}\right)$ and it follows that $\bar{\gamma} \in N_{\text {Aut } G}(H)=\operatorname{Inn} H$. Since $\operatorname{Inn} H \leq\left({ }^{*} H \times G^{*}\right)$, we may deduce that $\bar{\gamma}$ and hence $\gamma$ is contained in $\left({ }^{*} H \times G^{*}\right)$. This proves that $\left({ }^{*} H \times G^{*}\right)$ is self-normalizing in $\operatorname{Sym}(G)$.

We will exhibit some specific situations in which Lemma 5 can be applied.

(I) There are natural embeddings $M_{22} \leq M_{23} \leq M_{24} \leq \operatorname{Sym}(24)$ of the Mathieu groups wherein $M_{22}$ and $M_{23}$ are stabilizers of elements operated on by $M_{23}$ and $M_{24}$ respectively. Consider the two cases $H=M_{22}, G=M_{23}$ and $H=M_{23}$, $G=M_{24}$ where $H$ is regarded as a subgroup of $G$. Since $M_{22}, M_{23}$, and $M_{24}$ act 2transitively on $\{1, \ldots, n\}$ where $n=22,23$ and 24 respectively, they act primitively on them. It follows that $M_{22}$ is maximal in $M_{23}$ and $M_{23}$ is maximal in $M_{24}$. Lemma 5 implies that $\left({ }^{*} M_{23} \times M_{23}^{*}\right)$ and $\left({ }^{*} M_{23} \times M_{24}^{*}\right)$ are self-normalizing in $\operatorname{Sym}\left(M_{23}\right)$ and $\operatorname{Sym}\left(M_{24}\right)$ respectively. We deduce that the permutational wreath products $\left(S \mathrm{wr}\left({ }^{*} M_{22} \times M_{23}^{*}\right)\right)$ and $\left(S \mathrm{wr}\left({ }^{*} M_{23} \times M_{24}^{*}\right)\right)$ are complete p.n.a. groups, if $S$ is a complete nonabelian finite simple group. It should be emphasized that the group $\left({ }^{*} M_{22} \times M_{23}^{*}\right)$ is not complete since $M_{22}$ is not complete. This leads to the following proposition. 
PROPOSITON 6. Let $S$ be a complete nonabelian finite simple group and define $H_{1}=\left(S \mathrm{wr}\left({ }^{*} M_{22} \times M_{23}^{*}\right)\right)$ and $H_{2}=\left(S \mathrm{wr}\left({ }^{*} M_{23} \times M_{24}^{*}\right)\right)$. The groups $H_{1}$ and $H_{2}$ are complete p.n.a. groups but the composition series of $H_{1}$ contains a noncomplete factor.

(II) The symplectic groups of characteristic two give rise to self-normalizing p.n.a. subgroups of symmetric groups.

THEOREM 7. If $n \geq 7$, the projective symplectic groups $\operatorname{PSp}(2 n, 2) \equiv C_{n}(2)$ contain self-normalizing p.n.a. subgroups.

PROOF. Let $\left\{e_{-n}, \ldots, e_{-1}, e_{1}, \ldots, e_{n}\right\}$ be a basis for a vector space $V$ of dimension $2 n$ over $\mathrm{GF}(2)$. Define a skew-symmetric bilinear form on $V$ which operates on the basis of $V$ by $\left(e_{i}, e_{j}\right)=\delta_{j,-i}$ where $\delta_{i j}$ is the Kronecker delta. This form will be skew-symmetric since the field has characteristic two. The symplectic group $\operatorname{Sp}(2 n, 2)$ is the group of linear transformations of $V$ which preserve the bilinear form. Since the center of $\operatorname{Sp}(2 n, 2)$ consists of diagonal matrices, it is trivial. It follows that $\operatorname{Sp}(2 n, 2) \cong \mathrm{PSp}(2 n, 2) \equiv C_{n}(2)$, which is a complete nonabelian finite simple group.

Let $l$ and $m$ be distinct integers such that $l, m \geq 3$ and define $n=l+m$. Hence $n \geq 7$. Define $V_{1}=\left\langle e_{-l}, \ldots, e_{l-1}, e_{l}\right\rangle$ and $V_{2}=\left\langle e_{-n}, \ldots, e_{-l-1}, e_{l+1}, \ldots, e_{n}\right\rangle$. Notice that $\operatorname{dim} V_{1}=2 l$ and $\operatorname{dim} V_{2}=2 m$. It is clear that $V_{1}$ and $V_{2}$ are orthogonal subspaces such that $V=V_{1} \oplus V_{2}$. Define $W$ to be the subgroup of elements of $\operatorname{Sp}(2 n, 2)$ which leave $V_{1}$ and $V_{2}$ invariant. Let $F_{i}$ be the subgroup of elements of $W$ which act trivially on $V_{3-i}$ for $i=1$ and 2 . By restricting $F_{i}$ and the bilinear form to $V_{i}$, it is easily seen that $F_{1} \cong \mathrm{PSp}(2 l, 2)$ and $F_{2} \cong \mathrm{PSp}(2 m, 2)$. In addition, since $W=\left\langle F_{1}, F_{2}\right\rangle, F_{1} \cap F_{2}=I$, and $\left[F_{1}, F_{2}\right]=1$, we can deduce that $W=F_{1} \times F_{2}$. Since $l \neq m, W$ is a complete p.n.a. group, which by a well-known property of complete groups shows that $N_{S}(W)=W \times C_{S}(W)$ where $S=\operatorname{PSp}(2 n, 2)$.

We will complete the proof by showing that $C_{S}(W)=1$, so that $W$ is selfnormalizing in $S$. If we consider $S$ as a matrix group and define $E_{i, j}$ as the matrix with a 1 in the $(i, j)$ th position and zero elsewhere, then $\left(I+E_{i,-i}\right) \in W$ for all $i$. If $T \in C_{S}(W)$, then $E_{i,-i} T=T E_{i,-i}$. Writing $T=\left(t_{i, j}\right)$ and taking $(i, k)$ entries on each side, we obtain $t_{-i, k}=0$ for $k \neq-i$. This implies that $T$ is represented by a diagonal matrix and hence $T=1$. Thus $C_{S}(W)=1$.

We may apply Lemma 5 to the above examples to show that the group ${ }^{*}\left(C_{l}(2) \times C_{m}(2)\right) \times\left(C_{n}(2)\right)^{*}$ is a self-normalizing subgroup of $\operatorname{Sym}\left(C_{n}(2)\right)$. It follows that $S \operatorname{wr}\left({ }^{*}\left(C_{l}(2) \times C_{m}(2)\right) \times\left(C_{n}(2)\right)^{*}\right)$ is a complete p.n.a. group when $S$ is a complete nonabelian finite simple group. Similar techniques may be used with other Chevalley groups. Indeed, given a Chevalley group it is possible to construct subgroups of it by deleting selected points from its Dynkin diagram and considering the Chevalley group of the deleted diagram. Under suitable conditions, we may obtain a situation similar to the one given above.

In conclusion, we will consider yet another method of generating self-normalizing subgroups of a symmetric group.

LEMMA 8. Let $F$ be a complete finite group which contains a conjugacy clase $D$ such that $C_{F}(a)$ is self-normalizing for $a \in D$, and $C_{F}(D)=1$. Then there exists a self-normalizing subgroup $\bar{F}$ in $\operatorname{Sym}(D)$ which is isomorphic to $F$, namely the image of $F$ under the natural embedding of $F$ into $\operatorname{Sym}(D)$. 
Proof. Define $\sigma: F \rightarrow \operatorname{Sym}(D)$ as the map which takes $f$ into $\sigma_{f}$ where $\sigma_{f}$ is conjugation of the elements of $D$ by $f$. This map is monic, since $C_{F}(D)=1$. We will define $\bar{F}=\operatorname{Im} \sigma$.

If $\bar{\gamma} \in N_{\mathrm{Sym}(D)}(\bar{F})$, then $\bar{\gamma}=\sigma_{f} \gamma$ for some $f \in F$ and $\gamma \in C_{\mathrm{Sym}(D)}(\bar{F})$, since $F$ is complete. Choose an element $a \in D$. There exists $x \in F$ such that $(a) \gamma=a^{x}$. If $y \in C_{F}(a)$, then

$$
(a) \gamma=\left(a^{y}\right) \gamma=(a) \sigma_{y} \gamma=(a) \gamma \sigma_{y}=y^{-1}((a) \gamma) y
$$

and hence $C_{F}(a) \leq C_{F}\left(a^{x}\right)$. A similar argument yields $C_{F}\left(a^{x}\right) \leq C_{F}(a)$ and hence $C_{F}(a)=C_{F}\left(a^{x}\right)$. We may deduce that $x \in N_{F}\left(C_{F}(a)\right)=C_{F}(a)$; consequently (a) $\gamma=a$. It follows that $\left(a^{y}\right) \gamma=(a) \sigma_{y} \gamma=(a) \gamma \sigma_{y}=\left(a^{y}\right)$ for all $y \in F$ and this implies that $\gamma=1$. We have shown that $\bar{F}$ is self-normalizing in $\operatorname{Sym}(D)$.

If $F$ is a complete finite simple group with an element $a \neq 1$ such that $C_{F}(a)$ is self-normalizing, we can apply Lemma 8 because the condition $C_{F}(D)=1$ is automatically satisfied. The Mathieu group $M_{11}$ is an example of such a group.

PrOPOSITION 9. The Mathieu group $M_{11}$ is a complete nonabelian finite simple group with an element whose centralizer is self-normalizing.

PROOF. It is well known that $M_{11}$ is a complete nonabelian finite simple group. We may use the realization of $M_{11}$ as a subgroup of Sym(11) (Robinson [1, p. 202]) to show that the Sylow 2-subgroups of $M_{11}$ are of the form $S=\langle u, v| u^{8}=1=$ $\left.v^{2}, v^{-1} u v=u^{3}\right\rangle$. This group has center $\left\langle u^{4}\right\rangle$ of order two. Let $a=u^{4}$. It follows that $N_{M_{11}}(S) \leq C_{M_{11}}(a)$ and we may easily deduce that $C_{M_{11}}(a)$ is self-normalizing in $M_{11}$.

If $D$ is the conjugacy class containing $a$, then $|D|=\left|M_{11}: C_{M_{11}}(a)\right|=165$. It follows that Lemma 8 embeds $M_{11}$ in $\operatorname{Sym}(165)$ and the image is self-normalizing.

This paper is an excerpt from the author's doctoral dissertation at the University of Illinois (1983). The author would like to acknowledge the debt owed to his thesis advisor, Derek Robinson during the research and writing of his dissertation.

\section{REFERENCES}

1. D. J. S. Robinson, A course in the theory of groups, Springer-Verlag, New York, 1982.

Department of Mathematics, Michigan State University, East Lansing, MICHIGAN 48824

Current address: Department of Mathematics, The University of Alabama, Tuscaloosa, Alabama 35487-1416 